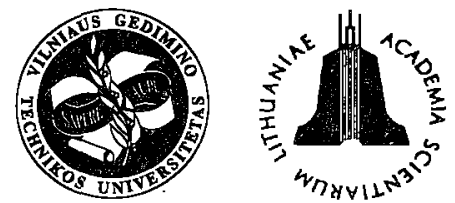

ISSN 1648-4142 TRANSPORT

http:/www.vtu.lt/english/editions

TRANSPORT - 2003, Vol XVIII, No 5, 224-228

\title{
NONLINEAR DYNAMIC ANALYSIS OF ROTATING PIPE CONVEYING FLUID BY THE FINITE ELEMENT METHOD
}

\author{
Marijonas Bogdevičius \\ Dept of Transport Technological Equipment, Vilnius Gediminas Technical University, \\ Plytinès g. 27, LT-2016 Vilnius, Lithuania \\ E-mail:marius@ti.vtu.lt, faksas:(370 5) 2745060; Tel. (370 5) 2744782 \\ Received 200305 28; accepted 20030901
}

\begin{abstract}
The rotating pipe conveying fluid is considered. Non-linear (geometrical non-linearity) equation of the motion of the pipe is derived using the finite element method. The equation of motion is derived based of the following assumptions: fluid is incompressible and inviscid; rotory inertia and shear deformation of the pipe are neglected. The sources of parametric vibration are: fluid velocity and pressure, angular speed of pipe. The possibility to solve a nonlinear response problem including sub- and/or super-harmonic using the finite element method is shown. Numerical results are derived considering the rotating contilevered pipe conveying fluid. All matrices and vectors of the finite element method are derived by the symbolic computation system Maple.
\end{abstract}

Keywords: Rotating pipe, fluid, vibration, dynamic response, finite element method.

\section{Introduction}

There is a great interest in the dynamic behavior of pipes conveying fluids. The problem of pipe conveying fluid is to be found in scientific literature [1-6], but the case is not largely considered when this problem is solved by the finite element method. So the problem of rotating pipes conveying fluids is of some interest. This problem is consideród by the nonlinear dynamic methods.

The purpose of this paper is to to present a finite element scheme for the determination dynamic instability (parametric resonance) of bending vibration of a rotating pipe conveying fluid.

\section{Formulation}

A rotating pipe of circle cross-section, along with the co-ordinate system employed, is shown in Fig 1. The pipe is assumed to rotate about $Z$-axis with uniform speed of $\Omega \mathrm{rad} / \mathrm{s}$. The material of the pipe is Hooken with a Young's modulus of elasticity $E$. Consider a pipe of length $L$ and flowing axially at velocity $V$.

Equation of motion is derived based on the following assumptions:

1. Fluid is inviscid and incompressible.

2. Rotary inertia and shear deformation of the pipe are neglected. glected.

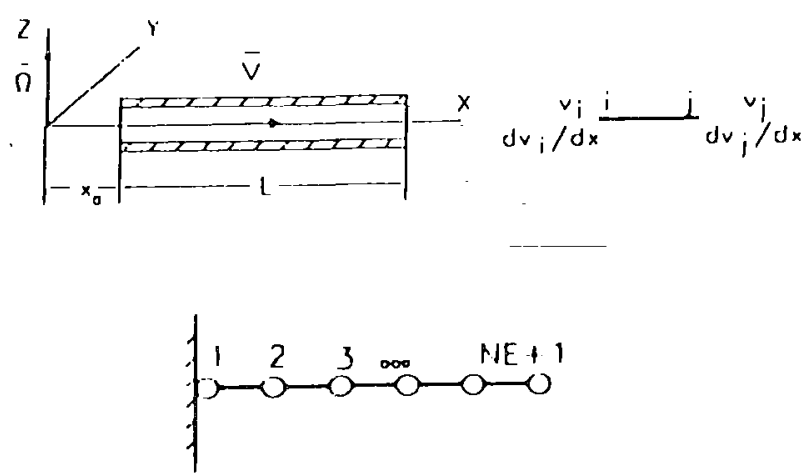

Fig 1. Rotating conveying fluid with co-ordinate system and finite element

The equation of motion can be derived based on either Hamilton's principle

$$
\int_{t} \delta(T-\Pi) d t+\int_{t} \delta A_{c} d t=0
$$

here $T$ and $\Pi$ are the kinetic and potential energies of system of pipe and fluid

$$
\begin{aligned}
& T=T_{p}+T_{f}, \\
& \Pi=\Pi_{p}+\Pi_{f} .
\end{aligned}
$$

The potential and kinetic energies for the pipes are

$$
\Pi_{p}=1 / 2 \int_{\nu} \sigma \varepsilon d v
$$




$$
T_{p}=1 / 2 \int_{\nu} \rho W^{2} d v
$$

where $\sigma, \varepsilon$ are the stress and the strain in the pipe respectively; $W$ is velocity of pipe; $\rho$ is the mass density of pipe.

Since the fluid is assumed to be incompressible, the potential energy of the fluid is zero, e.g.,

$$
\Pi_{f}=0 \text {. }
$$

The kinetic energy for the fluid is

$$
T_{f}=1 / 2 \int_{v} \rho_{f} W_{f}^{2} d v
$$

where $W_{f}$ is the flow velocity; $\rho_{f}$ is the mass density of fluid.

Large displacement relationships are considered. The deformed position of any point may be described in terms of $u$ and $v$. Assuming the Euler-Bernoulli theory, namely that plane sections before deformation will remain plane even after deformation, one may write

$$
u=-y d v / d x, v=v(x) .
$$

The total strain in the pipe is:

$$
\varepsilon=\varepsilon_{b}+\varepsilon_{c}+\varepsilon_{p},
$$

where $\varepsilon_{b}, \varepsilon_{c}, \varepsilon_{p}$ are strains of bending, centrifugal force and fluid pressure respectively.

The strain of bending of pipe is:

$$
\varepsilon_{b}=-y d^{2} v / d x^{2}+1 / 2(d v / d x)^{2},
$$

and $\sin (\theta) \approx d v d x$

$$
\cos (\theta) \approx 1-1 / 2 \theta^{2} \approx 1-1 / 2(d v / d x)^{2} .
$$

When the pipe is rotating with uniform angular velocity $\Omega$, it experiences a centrifugal force field defined by:

$$
\begin{aligned}
& F_{c}=\rho A \Omega^{2} \int_{x}^{L}\left(x+x_{0}\right) d x= \\
& \rho A \Omega^{2}\left[1 / 2\left(L^{2}-x^{2}\right)+x_{0}(L-x)\right] .
\end{aligned}
$$

In view of this, the pipe experiences a strain field given by:

$$
\varepsilon_{c}=F_{C} /(A E)
$$

where $A$ is cross section area of pipe.

Therefore the strain pressure of fluid in the pipe is:

$$
\varepsilon_{p}=a_{p} p / E,
$$

where $a_{p}$ - coefficient.

Using the above equations the expression for the potential energy $\Pi$ may be written as:

$$
\Pi=1 / 2 \int_{0}^{L}\left[\begin{array}{l}
E J\left(d^{2} v / d x^{2}\right)^{2}+1 / 4 E A(d v / d x)^{4}+ \\
F_{c}^{2} /(E A)+a_{p}^{2} A p^{2} / E+F_{c}(d v / d x)^{2}+ \\
2 a_{p} p F_{c} / E+a_{p} A p(d v / d x)^{2}
\end{array}\right]
$$

The pipe velocity may be written as:

$$
\{W\}=\{\Omega\} \times\{r\}+\{d U d t\}+\{V\},
$$

where $\{\Omega\}$ is vector of angular velocity, $\{\Omega\}^{T}=[0,0, \Omega]$ $\{r\}$ is radius-vector $\{r\}^{T}=\left[x_{0}+x, v, 0\right]$, and $\{U\}$ vector of displacement $\{U\}^{T}=[0, v, 0]$. yields:

Substituting the above equations into equation (15)

$$
\{W\}=\left[-\Omega v, \Omega\left(x_{0}+x\right)+d v d t, 0\right] .
$$

The flow velocity $W_{f}$ is

$$
\left\{W_{f}\right\}=\{\Omega\} \times\{r\}+\{d U d t\}+\{V\},
$$

where fluid velocity is:

$$
\{V\}=[V \cos (\theta), V \sin (\theta), 0], \operatorname{tg}(\theta)=d v / d x .
$$
yields:

Substituting the above equations into equation (17) $\left\{W_{f}\right\}=\left[V \cos (\theta)-\Omega \nu, \Omega\left(x_{0}+x\right)+d \nu / d t+V \sin (\theta), 0\right]$.

The pipe kinetic energy is:

$$
\begin{aligned}
& T_{p}=1 / 2 \int_{0}^{L} A \rho\left(\Omega^{2} v^{2}+\Omega^{2}\left(x_{0}+x\right)^{2}+(d v / d t)^{2}+\right. \\
& \left.2 \Omega\left(x_{0}+x\right) d v / d t\right) d x
\end{aligned}
$$

and the fluid kinetic energy is:

$T_{f}=1 / 2 \int_{0}^{L} A_{f} \rho_{f}\left(V^{2}+\Omega^{2} v^{2}(d v / d t)^{2}+\Omega^{2}\left(x_{0}+x\right)^{2}+\right.$

$2 V d v / d t \sin (\theta)+2 \Omega V\left(x_{0}+x\right) \sin (\theta)+$

$\left.2 \Omega\left(x_{0}+x\right) d v / d t-2 \Omega V v \cos (\theta)\right) d x$.

The work of centrifugal force may be written [6]:

$A_{c}=1 / 2 \int_{0}^{L}\left(\rho A+\rho_{f} A_{f}\right) \Omega^{2}\left[\left(x_{0}+x\right) \int_{0}^{x}(d v / d x)^{2} d x-v^{2}\right] d x$.

Applying the finite element scheme the pipe is divided into elements as shown in Fig 1. Two degrees of freedom per node, namely $v$ and $\theta=d v / d x$ are considered. In each finite element the displacement field is chosen as:

$$
\nu=[N\}\{q\},
$$

where $[N]$ is a matrix of shape functions and $\{q\}$ is the 
element displacement vector which depended on the time. Here $[N]$ is taken as:

$$
[N]=\left[N_{1} N_{2} N_{3} N_{4}\right],
$$

where

$$
\begin{aligned}
& N_{1}=1-3 \xi^{2}+2 \xi^{3} ; \\
& N_{2}=\xi-2 \xi^{2}+\xi^{3} ; \quad N_{3}=3 \xi^{2}-2 \xi^{3} ; \\
& N_{4}=\xi^{3}-\xi^{2}, \xi=x / L .
\end{aligned}
$$

\section{Nonlinear Dynamic Response Analysis}

In non-linear dynamic analysis it is well known that sub- and super-harmonic components of the response are also to be considered.

Using equation (22) in equations (14) and (19-21) one obtains the potential and kinetic energies of a finite element:

$$
\begin{aligned}
& \Pi^{(e)}=1 / 2\{q\}^{T}\left[K_{1}\right]\{q\}+1 / 2\{q\}^{T}\left[K_{2}\right\}\{q\}+ \\
& 1 / 2\{q\}^{T} \Omega^{2}\left[K_{3}\right]\{q\}+1 / 2\{q\}^{T} p\left[K_{4}\right]\{q\}, \\
& T^{(e)}=1 / 2\{q\}^{T} V^{2}\left[M_{s 13}\right\}\{q\}+1 / 2\{q\}^{T} \Omega^{2} . \\
& \left(\left[M_{s 1}\right]+\left[M_{1}\right]\right)+\{q\}-\{q\}^{T} \Omega V\left\{M_{s 10}\right\}+ \\
& 1 / 2\{q\}^{T} \Omega V\left[M_{s 6}\right\}\{q\} \\
& \{q\}^{T} \Omega V\left\{M_{s 7}\right\}+\{\dot{q}\}^{T}\left(\left[M_{s 1}\right]+\left[M_{1}\right]\{\dot{q}\}+\{\dot{q}\}^{T}\right. \\
& V\left[M_{s 2}\right\}\{q\}+\{\dot{q}\}^{T} \Omega\left(\left\{M_{s 3}\right\}+\left\{M_{3}\right\}\right)\{q\}, \\
& A_{c}^{(e)}=1 / 2\{q\}^{T} \Omega^{2}\left(\left[M_{s 12}\right]+\left[M_{12}\right]\right)\{q\}- \\
& 1 / 2\{q\}^{T} \Omega^{2}\left(\left[M_{s 1}\right]+\left[M_{1}\right]\right)\{q\}, \\
& {\left[K_{3}\right]=\int_{0}^{L}\left[B_{1}\right]^{T} F_{c}\left[B_{1}\right] d x,} \\
& {\left[K_{4}\right]=\int_{0}^{L}\left[B_{1}\right]^{T} a_{p} A p\left[B_{1}\right] d x,} \\
& {\left[M_{s 6}\right]=\int_{0}^{L}\left[B_{1}\right]^{T} \rho_{f} A_{f} v(x)\left(B_{1}\right) d x,} \\
& {\left[M_{13}\right]=1 / 4 \int_{0}^{L}\left[B_{1}\right]^{T} \rho_{f} A_{f} v(x)^{2}\left(B_{1}\right) d x .}
\end{aligned}
$$

The total energies of the system and the work done then become:

$$
\Pi=\sum_{e=1}^{N E} \Pi^{(e)}, T=\sum_{e=1}^{N E} T^{(e)}, A_{c}=\sum_{e=1}^{N E} A_{c}^{(e)} .
$$

Using equations (25) to (27) and Hamilton's principle (1) yields the governing equation of motion:

$[A]\{\ddot{q}\}+[B]\{\dot{q}\}+[C]\{q\}=\{D\}$,

where $[A]=\left[M_{s 1}\right]+\left[M_{1}\right],[B]=V\left(\left[M_{s 2}\right]-\left[M_{s 2}\right]^{T}\right)$,

$$
[C]=\left[K_{1}\right]+\left[K_{2}\right]+p\left[K_{4}\right]-V^{2}\left[M_{s 13}\right]-
$$

$$
\begin{aligned}
& \Omega^{2}\left(\left[M_{s 12}\right]+\left[M_{12}\right]\right)-\Omega V\left[M_{s 6}\right], \\
& \{D\}=\Omega V\left(\left[M_{s 7}\right]-\left[M_{s 10}\right]\right) .
\end{aligned}
$$

To facilitate their direct evaluation the element displacement is expressed as:

$$
q_{j}=\sum_{i=1}^{n_{1}} q_{j s i} \sin [(i-1) \omega t]+q_{j c i} \cos [(i-1) \omega t]+
$$

$$
\sum_{i=2}^{n_{2}} q_{j h s i} \sin \left[(\omega t / i)+q_{j h c i} \cos (\omega t) / i\right]
$$

$j=1, \ldots, 4$,

or $\{q\}=[S\} Q\}$,

$$
\begin{aligned}
& \{Q\}=\left\{q_{1 s 1}, \ldots, q_{1 s n_{1}}, q_{1 c 1}, \ldots, q_{1 c n_{1}}, q_{1 h s 2}, \ldots\right. \\
& q_{1 h s n_{2}}, q_{1 h c 2}, \ldots, q_{1 h c n_{2}}, \ldots, q_{4 s 1}, \ldots, q_{4 s n_{1}}, q_{4 c 1}, \ldots
\end{aligned}
$$$$
\left.q_{4 c n_{2}}, q_{4 h s_{2}}, \ldots, q_{4 h s n_{2}}, q_{4 h c_{2}}, \ldots, q_{4 h c n_{2}}\right\}^{T} \text {. }
$$$$
[S]=\operatorname{diag}([Z]),
$$$$
[Z]=\left[0, \sin (\varphi), \ldots, \sin \left(\left(n_{1}-1\right), 1, \cos (\varphi), \ldots, \cos \left(n_{1}-1\right) \varphi\right),\right.
$$$$
\left.\left.\sin (\varphi / 2), \ldots, \sin \left(\varphi / n_{2}\right), \ldots, \cos (\varphi / 2), \ldots, \cos \left(\varphi / n_{2}\right)\right)\right],
$$

$\varphi=\omega t$

Thus one has $v=[N]\{q\}=[N][S\}\{Q\}$.

Substituting expression (34) in the element energy expressions gives:

$$
\begin{aligned}
& \Pi=1 / 2\{Q\}^{T}\left[A_{0}\right]\{Q\}, \\
& T=1 / 2\{Q\}^{T}\left[A_{1}\right]\{Q\}+\{Q\}^{T}\left[A_{2}\right], \\
& A_{C}=1 / 2\{Q\}^{T}\left[A_{3}\right]\{Q\},
\end{aligned}
$$


$\left[A_{1}\right]=\int_{0}^{T} \int_{0}^{L}[S]\left(V^{2}\left[M_{s 13}\right]+\Omega^{2}\left(\left[M_{s 1}\right]+\left[M_{1}\right]\right)+\right.$

$\left.\Omega V\left[M_{s 6}\right]\right)[S] d x d t+\int_{00}^{T} \int_{0}^{L}\left(\left[C_{1}\right]^{T}+\left(\left[M_{s 1}+M_{1}\right]\right)\right.$

$\left.\left[C_{1}\right]+\left[C_{1}\right]^{T} V\left[M_{s 2} \mathbb{I}\right]\right) d x d t$

$\left[A_{2}\right]=\int_{00}^{T L} \int\left([s]^{T}\left(\left\{M_{s 7}\right\}-\left\{M_{s 10}\right\}\right)+\left[C_{1}\right] \Omega\left(\left\{M_{s 3}\right\}+\left\{M_{3}\right\}\right)\right) d x d t$,

$\left.\left[A_{3}\right]=\int_{00}^{T L} \int^{T}[S]\left(\Omega^{2}\left(\left[M_{s 12}\right]+\left[M_{12}\right]\right)-\Omega^{2}\left(\left[M_{s 1}\right]+\left[M_{1}\right]\right)\right) S\right] d x d t$,

$\left[C_{1}\right]=d[S] / d t, \quad T=2 \pi / w$.

All matrices and vectors of the finite element method are derived using symbolic computation systems Maple [6-8].

Applying Hamilton's principle then gives the governing equation of motion as:

$$
[K(Q)\}\{Q\}=\{P(Q)\},
$$

where

$$
\begin{aligned}
& {[K(Q)]=\int_{0}^{T}[S]\left(\left[K_{1}\right]+\left[K_{2}\right]+p\left[K_{4}\right]-V^{2}\left[M_{s 13}\right]-\right.} \\
& \left.\Omega V\left[M_{s 6}\right]-\Omega^{2}\left(\left[M_{s 12}\right]+\left[M_{12}\right]\right)\right)[S] d x d t \\
& \int_{0}^{T}\left[C_{1}\right]^{T}\left(\left(\left[M_{s 1}\right]+\left[M_{1}\right]\right)\left[C_{1}\right]-V\left[M_{s 2} \mathbb{I} S\right]\right) d t
\end{aligned}
$$$$
\{P(Q)\}=\int_{0}^{T}[S] \Omega V\left(\left\{M_{s 7}\right\}-\left\{M_{s 10}\right\}\right)+
$$$$
\left[C_{1}\right]^{T} \Omega\left(\left\{M_{s 3}\right\}+\left\{M_{3}\right\}\right) d t \text {. }
$$

After applying the boundary conditions, equations (39) are solved iteratively for the evaluation of $\{Q\}$ using Newton method.

\section{Numerical Example}

For numerical studies a pipe of a circular cross-section with the inner diameter $d=0,008 \mathrm{~m}$, thickness $h=0,001 \mathrm{~m}$ and length $L=1 \mathrm{~m}$ is considered. The material of the pipe was taken steel with $E=2,1 \cdot 10^{11} \mathrm{~Pa}$.

The boundary conditions for a cantilevered pipe are:

$x=0, v=0, \partial v \partial x=0$,

$$
x=L, \partial^{2} v / \partial x^{2}=0, \partial^{3} v / \partial x^{3}=0 .
$$

Figs 2-4 show the one-third sub-harmonic of the rotating cantilevered pipe conveying fluid dependence on the frequency and the fluid pressure, flow velocity and the angular velocity respectively.

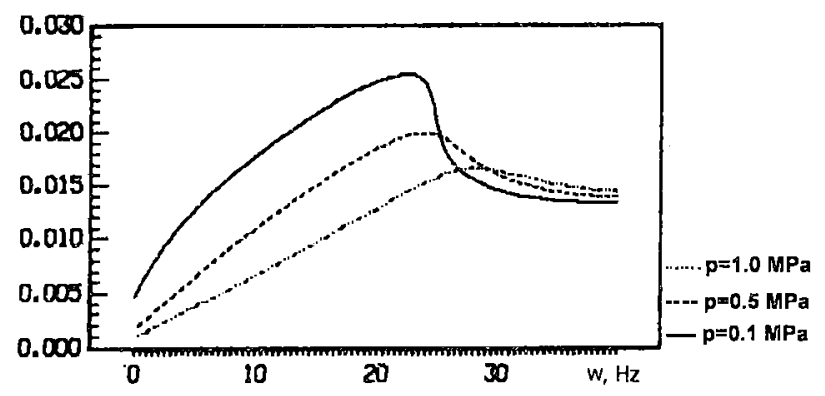

Fig 2. The one-third subharmonic $q_{3 h s}(x=L) / L$ as function of the frequency $\omega$ and the fluid pressure $p$ : $L=1 \mathrm{~m}, x_{0}=0 \mathrm{~m}, d_{i n}=0,008 \mathrm{~m}, d=0,010 \mathrm{~m}$,

$$
E=2,1 \cdot 10^{11} \mathrm{~Pa}
$$

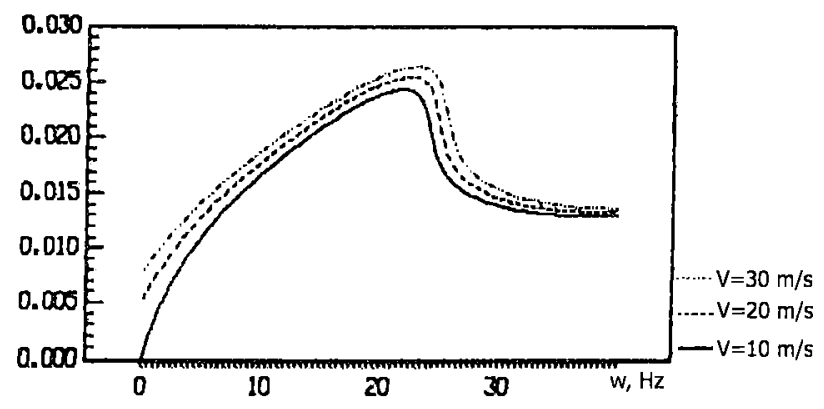

Fig 3. The one-third subharmonic $q_{3 h s}(x=L) / L$ as function of the frequency $\omega$ and the flow velocity $v$ : $L=1 \mathrm{~m}, x_{0}=0 \mathrm{~m}, d_{\text {in }}=0,008 \mathrm{~m}, d=0,010 \mathrm{~m}$, $E=2,1 \cdot 10^{11} \mathrm{~Pa}$

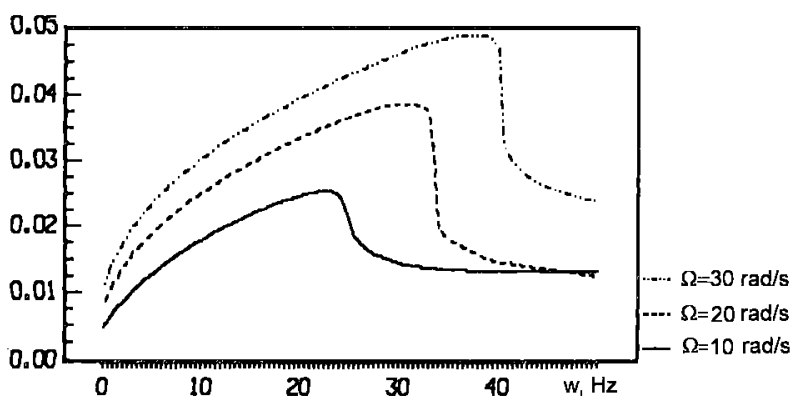

Fig 4. The one-third subharmonic $q_{3 h s 3}(x=L) / L$ as function of the frequency $\omega$ and angular speed $\Omega: L=1 \mathrm{~m}$, $x_{0}=0 \mathrm{~m}, d_{i n}=0,008 \mathrm{~m}, d=0,010 \mathrm{~m}, E=2,1 \cdot 10^{1} \mathrm{~Pa}$

\section{Conclusions}

The nonlinear dynamic analysis of the rotating pipe conveying fluid is presented. The one-third sub-harmonic amplitudes of the rotating cantilevered pipe conveying fluid are obtained. The one-third sub-harmonic ampli- 
tudes depend on fluid pressure, flow velocity and angular velocity.

\section{References}

1. Ahmadi, G.; Sater, M. Stability of a pipe carrying timedependent flowing fluid. Journal of the Franklin Institute, 305 (1), 1978, p. 1-9.

2. Chen, S.; Jendrzejczyk, A. Stability of tubes conveying fluid. Argone National lab. ANL-84-5, 1984.

3. Chen, S. Flow - induced vibration of circular cylindrical structures. Springer - Verlag, 1987.

4. Paliūnas, V; Paliūnienè, A. Free vibrations of bars in liquid. (Strypu svyravimai skystyje).Vilnius: Mokslas, 1978 (in Lithuanian).
5. Bogdevičius, M. Linear Dynamic Analysis of Rotating Pipe Conveying Fluid by Finite Element. Lithuanian Journal of Computational Mechanics. Vilnius, 1993, Vol, 33, p. 5664.

6. Aladjev, V:; Bogdevicius, M. Solution of Physical, Technical and Mathematical Problems with Maple V (Решение физико-технических и математических задач с пакетом Maple V). Vilnius, 1999. 686 p. (in Russian).

7. Aladjev, V.; Bogdevicius, M. Maple 6: Solution of the Mathematical, Statistical and Engineering-physical Problems (Maple 6: Решение математических, статистических и инженерно-физических задаq). Moscow, 2001. 824 p (in Russian).

8. Aladjev, V.; Bogdevicius, M.; Prentkovskis, O. New Software for Mathematical Package Maple of Releases 6, 7 and 8. Vilnius: Technika, 2002. 404 p. 